\title{
LOW TEMPERATURE PROPERTIES OF NTD GE: BEST CHOICE FOR CUORE EXPERIMENT
}

\author{
EDOARDO PASCA, EMILIANO OLIVIERI, GUGLIELMO VENTURA \\ Physics Department University of Firenze and INFM Unity of Firenze \\ MARCO BARUCCI, LARA RISEGARI \\ Mechanics Department University of Firenze and INFN Section of Firenze \\ JEFFREY BEEMAN \\ Lawrence Berkeley National Laboratory, University of California, Berkeley
}

\begin{abstract}
A new series of NTD Ge thermistors has been produced by the Lawrence Berkeley National Laboratory in the framework of the CUORE (Cryogenic Underground Observatory for Rare Events) experiment. The electrical characteristics of the new materials have been measured at the Departement of Physics of the University of Florence. The goal of this study was the choice of the best type of thermistor to use in the CUORE experiment. We report also the electrical resistivity, electron specific heat and electron-phonon decoupling of the NTD31 Ge material selected for CUORICINO (single CUORE module, which has become operative at the end of 2002).
\end{abstract}

\section{Introduction}

The main goal of CUORE (Cryogenic Underground Observatory for Rare Events) is the detection of a neutrinoless double $\beta$ decay $^{1}$. The CUORE detector will consist of an array of 1000 closely packed low temperature $\mathrm{TeO}_{2}$ bolometers. The array will consist of 25 towers containing 10 cells of 4 bolometers each.

The investigated decay is ${ }^{130} \mathrm{Te} \rightarrow{ }^{130} \mathrm{Xe}+2 e^{-}$(transition energy 2528 $\mathrm{keV})$. The decay should take place in the $\mathrm{TeO}_{2}$ absorbers. The isotopical abundance of ${ }^{130} \mathrm{Te}$ is $34 \%$ of total Te atoms ${ }^{2}$.

Each bolometer consists of a $\mathrm{TeO}_{2}$ absorber of $5 \times 5 \times 5 \mathrm{~cm}^{3}$ dimensions and a thermal sensor (a chip of Neutron Transmutation Doped Germanium of low specific heat ${ }^{3}$ and high sensitivity).

The CUORICINO experiment, consisting of a single CUORE tower with 
$445 \times 5 \times 5 \mathrm{~cm}^{3}$ and $183 \times 3 \times 6 \mathrm{~cm}^{3}$ absorbers and NTD31 Germanium thermistor as thermal sensor, is presently running in the Laboratori Nazionali del Gran Sasso.

In this paper we report on the production of 9 new types of NTD Ge for the CUORE project (numbered in ascending order from 33 to 38 , and 33B, 34B, 35B; the latter three being series 33-35 that have received a second neutron irradiation); we investigated the properties of these new NTD Ge sensors, as well as those of NTD 31, aiming to select the best one for CUORE. Furthermore, for NTD 31 we report electronic specific heat and electron-phonon decoupling.

\section{NTD Germanium}

NTD Germanium is produced by irradiating an ultrapure Ge crystal by means of a flux of thermal neutrons. Ge nuclei capture the neutrons and form various radioactive Ge isotopes, which subsequentely decay into As, Se and $\mathrm{Ga}^{4}$. Due to the isotopical composition of Ge and to the neutron cross section, the resulting doping is of p-type. The neutron capture cross section for Ge nuclei is sufficiently low that the neutron flux can be considered constant through all the Ge crystal, resulting in a very uniform distribution of dopants in the crystal.

Such a doped semiconductor, at low temperatures, has a resistivity dominated by Variable Range Hopping effect ${ }^{5}$ :

$$
\rho(T)=\rho_{0} \exp \left(\frac{T_{0}}{T}\right)^{\alpha}
$$

where $\rho_{0}, T_{0}$ and $\alpha$ are constants. $\alpha$ depends on the shape of the density of states of the charge carriers at the Fermi surface. For NTD Ge it is believed that the density of states goes to zero at the Fermi surface as a parabola, resulting in a $\alpha=0.5^{5}$. $T_{0}$ is related to the doping level of the thermistor, and it increases as doping decreases; $\rho_{0}$ depends on the intrinsic properties of the Ge lattice and on stresses applied to the sample, and it does not depend on the doping level ${ }^{4}$.

At very low temperature, the phenomenon of electron-phonon decoupling, which is well known in metals ${ }^{6}$, is present in NTD Ge. Electronphonon decoupling is due to the fact that electrons, do not have enough states available to excite a phonon, or viceversa. An empirical model (Hot Electron Model ${ }^{4,7}$ ) describes the electron and the phonon systems as com- 
municating via a thermal conductance $G_{e-p}$, which represents the decoupling between the two systems.

Considering a degenerate electron gas, the number of states available at the Fermi surface increases as $N^{\frac{1}{3}}, N$ being the total number of electrons in the gas. In this rough approximation one would expect the $G_{e-p}$ in NTD Ge to increase with the increase of doping (i.e. decrease of $T_{0}$ ).

Electron-phonon decoupling tends to worsen the detector performance in the low temperature range, since together with the electron heat capacity it changes the pulse shape and rise time.

\section{Experiments and Results}

\subsection{Sample Production}

Each pure Ge sample was irradiated by a flux of thermal neutrons and then annealed at about $400^{\circ} \mathrm{C}$ for six hours in order to "repair" damage in the crystal structure. Electrical contacts were made by heavily doping two sides of the sample with $\mathrm{B}^{+}$ions $\left(3 \cdot 10^{14} \mathrm{~cm}^{-2}\right)$ to a depth of about $200 \mathrm{~nm}$. A layer of $\mathrm{Pd}$ (about $20 \mathrm{~nm}$ ) and one of $\mathrm{Au}$ (about $400 \mathrm{~nm}$ ) are sputtered onto the two surfaces. The sample is then annealed at $250^{\circ} \mathrm{C}$ for one hour.

\subsection{Electrical resistivity}

Low temperature resistivity of the new NTD samples was measured in a dilution refrigerator, in the temperature range $26-200 \mathrm{mK}$.

Each sample (chip of about $3 \times 3 \times 1 \mathrm{~mm}^{3}$ ) was glued on a copper holder in thermal contact with the mixing chamber. Electrical insulation and good thermal contact between holder and sample was obtained by interposing a thin foil of mylar $(4 \mu \mathrm{m})$. The electrical contacts were made with two thin gold wires $(50 \mu \mathrm{m}$ in diameter, about $2 \mathrm{~cm}$ long) ball-bonded to each $\mathrm{Au}$ surface. 2 manganine wires were soldered to each gold wire. An LR-700 $\mathrm{AC}$ resistance bridge was used to measure the resistance of the sample. A calibrated $\mathrm{RuO}_{2}$ thermometer and an AVS-47 AC bridge were used to measure the temperature of the samples. Each electrical lead to the experiment was RF filtered on the top of the cryostat.

In Table 1 we report the results of the measurements carried out on the NTD samples. 
Table 1. Data of the 9 NTD series produced for CUORE, and of NTD 31; doping is the compensate doping: \#Ga-\#As-2\#Se.

\begin{tabular}{|c|c|c|c|c|}
\hline $\begin{array}{c}\text { NTD } \\
\text { family }\end{array}$ & $\begin{array}{c}\text { Dimensions } \\
{\left[\mathrm{mm}^{3}\right]}\end{array}$ & $\begin{array}{c}T_{0} \\
{[\mathrm{~K}]}\end{array}$ & $\begin{array}{c}R_{0} \\
{[\Omega]}\end{array}$ & $\begin{array}{c}\text { Doping } \\
{\left[\mathrm{cm}^{-3}\right]}\end{array}$ \\
\hline 33 & $2.9 \times 1.6 \times 6$ & $12.44 \pm 0.08$ & $0.328 \pm 0.001$ & $5.0 \mathrm{E} 16$ \\
34 & $2.9 \times 1.6 \times 6$ & $10.88 \pm 0.05$ & $0.278 \pm 0.001$ & $5.3 \mathrm{E} 16$ \\
35 & $2.9 \times 1.6 \times 6$ & $9.27 \pm 0.08$ & $0.342 \pm 0.001$ & $5.6 \mathrm{E} 16$ \\
36 & $2.9 \times 1.6 \times 6$ & $7.07 \pm 0.05$ & $0.208 \pm 0.001$ & $5.9 \mathrm{E} 16$ \\
37 & $2.9 \times 1.6 \times 6$ & $5.41 \pm 0.07$ & $0.281 \pm 0.001$ & $6.0 \mathrm{E} 16$ \\
38 & $2.9 \times 1.6 \times 6$ & $4.66 \pm 0.06$ & $0.283 \pm 0.001$ & $6.0 \mathrm{E} 16$ \\
31,8 & $2.9 \times 1.6 \times 6$ & 4.1 & 0.164 & $6.6 \mathrm{E} 16$ \\
$33 \mathrm{~B}$ & $3 \times 3 \times 1$ & $4.25 \pm 0.04$ & $0.830 \pm 0.001$ & $6.2 \mathrm{E} 16$ \\
$34 \mathrm{~B}$ & $3 \times 3 \times 1$ & $4.23 \pm 0.04$ & $0.835 \pm 0.001$ & $6.4 \mathrm{E} 16$ \\
$35 \mathrm{~B}$ & $3 \times 3 \times 1$ & $3.07 \pm 0.03$ & $0.949 \pm 0.001$ & $6.8 \mathrm{E} 16$ \\
\hline
\end{tabular}

\subsection{Specific heat and Electron-Phonon decoupling of NTD31}

The Debye temperature of Ge is $370 \mathrm{~K}^{9}$. Hence, at very low temperature, its specific heat ${ }^{3}$ is due to the charge carriers: $c(T)=\gamma T$ with $\gamma=1.05$. $10^{-6} \mathrm{~J} \mathrm{~K}^{-2} \mathrm{~cm}^{-3}$. The total heat capacity of a NTD 31 thermal sensor (the dimensions of the thermal sensor are $3 \times 3 \times 1 \mathrm{~mm}^{3}$ ) at $10 \mathrm{mK}$ is about $4 \%$ of that of the absorber ${ }^{10}$.

We have measured the electron-phonon decoupling in NTD $31 \mathrm{Ge}^{8}$; this effect becomes important below a temperature of $T_{d} \approx 30 \mathrm{mK}$. We expect this temperature $T_{d}$ to decrease with increase of doping, but further measurements on different types of NTD Ge are necessary.

\section{Conclusions}

In the frame of the CUORE experiment, 9 new NTD Ge materials have been produced. On the basis of the electrical characteristics $\left(T_{0} \approx 3 \mathrm{~K}\right)$ the NTD 35B seems to be the best choice for CUORE.

Further measurements on charge carriers specific heat and electronphonon decoupling will be necessary before the final choice.

\section{References}

1. Arnaboldi C. et al., Astroparticle Physics, In Press, Corrected Proof, Available online 9 August 2003

2. Pirro S. et al., Nucl. Instr. Meth. in Phys. Res. A, 44471 (2000)

3. Pedretti M. et al., Physica B Condensed Matter, 329, 1614 (2003)

4. Wang N. , Ph.D. Thesis, University of California at Berkeley, 1991 
5. Shklovskii B.I., Efros A.L., Electronic Properties of doped semiconductors, Springer-Verlag (1984)

6. Pobell F., "Matter and Methods in low temperature physics", SpringerVerlagh Berlin 1986

7. Wang N. et al., J. Phys. Rev. B 41 N.6, 3761 (1990)

8. Pasca E. et al., Proc. ICATPP7, p.684 World Scientific 2002

9. Keesom P.H. and Seidel G., Phys. Rev. 113, 1959

10. Barucci M. et al., J. of Low Temp. Physiscs 123 (5-6): 303-314, June 2001 